\title{
DIFFERENTIABILITY OF THE EXPONENTIAL OF A MEMBER OF A NEAR-RING
}

\author{
J. W. NEUBERGER ${ }^{1}$
}

ABSTRACT. Suppose $S$ is a Banach space and $K$ is the near-ring of all zero preserving Lipschitz transformations from $S$ to $S$. It is shown that all exponentials of members of $K$ have certain differentiability properties. This leads to the fact that no neighborhood of the identity transformation is filled with exponentials of members of $K$.

It is known ([1], [3] or [2]) that if $K$ is a Banach algebra with identity $I$ and $T$ is an element of $K$ such that $|T-I|<1$, then $T=\operatorname{Exp} A$ for some $A \in K$. Here $\operatorname{Exp} A=\lim _{n \rightarrow \infty}(I+(1 / n) A)^{n}$. From this fact it follows easily that the identity component of $K$ is precisely the set of all finite products of exponentials of members of $K$.

One purpose of this note is to show that in a near-ring of zero preserving Lipschitz transformations on a Banach space, it no longer holds that all elements in some neighbor hood of $I$ are exponentials. It was shown in [2] that for such near-rings the finite products of exponentials are dense in the identity component. The results of this note indicate that [2] cannot be improved in a certain direction.

Denote by $S$ a real Banach space and by $K$ a near-ring of zero preserving Lipschitz transformations from $S$ to $S$. Precisely, $K$ is a collection of transformations from $S$ to $S$ such that:

(1) if each of $T$ and $V \in K$ and $c$ is a number, then $T+V, T V$ and $c T \in K$;

(2) if $T \in K$, there is a smallest number $|T|$ (called the norm of $T$ ) such that $\|T x-T y\| \leq|T|\|x-y\|$ for all $x, y \in S$;

(3) $T 0=0$;

(4) $I$, the identity transformation on $S$, is in $K$;

(5) $K$ is complete in the sense that if $T$ is a transformation from $S$ to $S$ such that (2) holds and $\left\{T_{i}\right\}_{i=1}^{\infty}$ is a sequence of elements of $K$ which converges to $T$ uniformly on each bounded subset of $S$, then $T \in K$.

If $A \in K$ then $\operatorname{Exp} A$ denotes the element $L$ of $K$ such that $L x=$

Received by the editors December 31, 1973.

AMS (MOS) subject classifications (1970). Primary $47 \mathrm{H} 99$.

Key words and phrases. Identity component, normed near-ring exponential.

1 Supported in part by an NSF grant. 
$\lim _{n \rightarrow \infty}(I+(1 / n) A)^{n} x$ for all $x \in S$. Properties of this exponential function are listed in [2].

Theorem 1. Suppose each of $A$ and $B$ is in $K$ and $(\operatorname{Exp} t A)(\operatorname{Exp} s B)=$ $(\operatorname{Exp} s B)(\operatorname{Exp} t A)$ for all numbers $t$ and $s$. If $x \in S, t$ is a number and $B x \neq$ 0 , then $\operatorname{Exp} t A$ is G-differentiable at $x$ in the direction $B x$ and

$$
\left(D_{B x} \operatorname{Exp} t A\right) x=(B \operatorname{Exp} t A) x \text { for all numbers } t .
$$

Proof. From [2, Lemma 0 (iv)], it follows that if $Q \in K$ and $x \in S$, then $\|(\operatorname{Exp} \delta Q) x-(I+\delta Q) x\|=\|(\operatorname{Exp} \delta Q) x-(x+\delta Q x)\|=o(\delta)$ as $\delta \rightarrow 0$.

Therefore for $x \in S$ and $B x \neq 0$,

$$
\begin{aligned}
\|[(\operatorname{Exp} t A)(x+\delta B x)-(\operatorname{Exp} t A) & x]-\delta(B \operatorname{Exp} t A) x \| \\
= & \|(I+\delta B)(\operatorname{Exp} t A) x-(\operatorname{Exp} t A)(I+\delta B) x\| \\
\leq & \|(I+\delta B)(\operatorname{Exp} t A) x-(\operatorname{Exp} \delta B)(\operatorname{Exp} t A) x\| \\
& +\|(\operatorname{Exp} t A)(\operatorname{Exp} \delta B) x-(\operatorname{Exp} t A)(I+\delta B) x\| \\
\leq & \|(\operatorname{Exp} \delta B)(\operatorname{Exp} t A) x-(I+\delta B)(\operatorname{Exp} t A) x\| \\
& +e^{|t A|}\|(\operatorname{Exp} \delta B) x-(I+\delta B) x\| \\
= & o(\delta) \text { as } \delta \rightarrow 0 .
\end{aligned}
$$

So, $\|[(\operatorname{Exp} t A)(x+\delta B x)-(\operatorname{Exp} t A) x]-\delta(B \operatorname{Exp} t A) x\|=o(\delta)$ as $\delta \rightarrow 0$. But this is precisely the statement that $\operatorname{Exp} t A$ is $G$-differentiable at $x$ in the direction $B x$ and that $\left(D_{B x} \operatorname{Exp} t A\right) x=(B \operatorname{Exp} t A) x$.

Corollary. If $A \in K, t$ is a number and $x \in S$, then $\left(D_{A x} \operatorname{Exp} t A\right) x=$ $(A \operatorname{Exp} t A)_{x}$

This follows from Theorem 1 since

$$
(\operatorname{Exp} t A)(\operatorname{Exp} s A)=\operatorname{Exp}(t+s) A=(\operatorname{Exp} s A)(\operatorname{Exp} t A)
$$

for all numbers $t$ and $s$.

Theorem 2. Suppose $K$ is the near-ring of all zero preserving Lipschitz transformations on $S$. Then no neighborhood of $I$ is filled with exponentials of members of $K$.

Proof. Suppose $\epsilon>0$. Pick $y_{\epsilon} \in S$ such that $\left\|y_{\epsilon}\right\|=\epsilon$ and define

$$
T_{\epsilon} z= \begin{cases}z & \text { if }\left\|z-y_{\epsilon}\right\| \geq \epsilon, \\ z-\left(\epsilon-\left\|z-y_{\epsilon}\right\|\right) y_{\epsilon} & \text { if }\left\|z-y_{\epsilon}\right\| \leq \epsilon .\end{cases}
$$


It may be verified that $\left|T_{\epsilon}-I\right|=\epsilon$, and if $x \in S,\|x\| \neq 0$, a is a number different from 0 so that $|a|<\epsilon /\|x\|$, then

$$
(1 / \alpha)\left[\left(T_{\epsilon}-I\right)\left(y_{\epsilon}+\alpha x\right)-\left(T_{\epsilon}-I\right) y_{\epsilon}\right]=(|\alpha| / \alpha)\|x\| y_{\epsilon} .
$$

Since $\lim _{\alpha \rightarrow 0}(|\alpha| / \alpha) y_{\epsilon}$ does not exist, it follows that $T_{\epsilon}-I$ (and hence $\left.T_{\epsilon}\right)$ is not $G$-differentiable in any direction at $y_{\epsilon}$.

Suppose now that $T_{\epsilon}=\operatorname{Exp} A$ for some $A \in K$. Then $A y_{\epsilon} \neq 0$, since if it were zero, then

$$
(1-\epsilon) y_{\epsilon}=T_{\epsilon} y_{\epsilon}=(\operatorname{Exp} A) y_{\epsilon}=\lim _{n \rightarrow \infty}(I+(1 / n) A)^{n} y_{\epsilon}=y_{\epsilon},
$$

a contradiction.

By the Corollary, $T_{\epsilon}=\operatorname{Exp} A$ is $G$-differentiable in the direction $A y_{\epsilon}$, a contradiction to the fact established above that $T_{\epsilon}$ is not $G$-differentiable at $y_{\epsilon}$ in any direction. Hence the assumption that $T_{\epsilon}=\operatorname{Exp} A$ for some $A$ $\epsilon K$ is false and the theorem is established.

The problem is left open of characterizing those near-rings of zero preserving Lipschitz transformations on $S$ which do have a neighborhood of the identity filled with exponentials of members of the nearoring. This is a special case of the following more general problem:

Denote by $K$ the near-ring of all zero preserving Lipschitz transformations on a Banach space $S$. Denote by $K^{\prime}$ the identity component of $K$. Characterize those subgroups of $K^{\prime}$ which have a neighborhood of $I$ which is filled with exponentials of members of $K$.

A closely related problem is that of finding a necessary and sufficient condition for an element $T$ of $K(|T-I|<1)$ to be an exponential of some member of $K$.

\section{REFERENCES}

1. M. Nagumo, Einige analytische Untersuchunger in linearen metrischen Ringen, Japan J. Math. 13 (1936), 61-80.

2. J. W. Neuberger, Toward a characterization of the identity component of rings and near-rings of continuous transformations, J. Reine Angew. Math. 238 (1969), 100-104. MR 40 \#3384.

3. J. von Neumann, Über die analytischen Eigenschaften von Gruppen linearer Transformationen und ihrer Darstellungen, Math. Z. 30 (1929), 3-42. TUCKY 40506

DEPARTMENT OF MATHEMATICS, UNIVERSITY OF KENTUCKY, LEXINGTON, KEN-

Current address: Department of Mathematics, Emory University, Atlanta, Georgia 30322 